\title{
SINGLE BUNCH LONGITUDINAL MEASUREMENTS OF THE CORNELL ELECTRON-POSITRON STORAGE RING WITH THE SUPERCONDUCTING RF CAVITIES*
}

\author{
R. Holtzapple ${ }^{\#}$, D. Rice, Cornell University, Ithaca, New York
}

\begin{abstract}
As part of the Phase III luminosity upgrade, the Cornell Electron-Positron Storage Ring (CESR) RF system has been upgraded from normal conducting (NRF) to superconducting (SRF) cavaties. The new superconducting cavities change the overall impedance of CESR and thus change the longitudinal bunch distribution. Measurements of the single bunch longitudinal distribution have been made using a streak camera on CESR with the SRF cavities present. These measurements are compared with earlier measurements on CESR with the NRF cavities. The CESR vacuum chamber impedance is determined from the current dependence of the single bunch charge distribution.
\end{abstract}

\section{INTRODUCTION}

The Cornell Electron-Positron Storage Ring (CESR) is a $768 \mathrm{~m}$ circumference e+e- storage ring operating at a beam energy between 4.7 and $5.6 \mathrm{GeV}$ to study decays of the B meson and the narrow Y resonances. Between 1997 and 1999, the CESR RF system was upgraded with the installation of superconducting RF cavities (referred to as SRF cavities)[1]. Four single-cell niobium superconducting RF cavities, designed to support 1 ampere of stored current at a peak luminosity of $1.7 \times 10^{33} \mathrm{~cm}^{-2} \mathrm{~s}^{-1}$, now provide RF acceleration in CESR. Previously the storage ring relied on four 5-cell normal conducting copper RF accelerating cavities (referred to as NRF cavities). With the NRF cavities, CESR could collide a maximum total current of $350 \mathrm{~mA}$ and achieve a peak luminosity of $4.5 \times 10^{32} \mathrm{~cm}^{-2} \mathrm{~s}^{-1}$. A longitudinal coupled bunch instability, caused by higher-order modes in the NRF cavities, limited higher current collisions and higher luminosity. To overcome the limitations of the 5cell normal conducting cavities, the single cell superconducting cavities have the advantage of: 1) Operating at higher gradient per cell to reduce the overall number of cells from 20 to 4,2 ) having a cell shape that strongly damps higher order modes, 3 ) a $24 \mathrm{~cm}$ diameter beam tube that reduces the overall impedance of the cavities. These improvements reduce bunch-to-bunch coupling, thus raising the threshold currents of multibunch instabilities

The longitudinal distributions of charge within the bunches with both NRF and SRF cavities have been measured and compared to determine the change in the overall vacuum chamber impedance in CESR.

\footnotetext{
*This work was supported by the National Science Foundation.

\# Present e-mail:RLH@SLAC.Stanford.edu.
}

The measurements with NRF cavities were done using a streak camera borrowed from the Stanford Linear Accelerator Center [2]. The measurements presented here (with SRF cavities) were made with a different unit since acquired by Cornell. For both sets of measurements the camera model is Hamamatsu C1587 with a M1952 highspeed streak unit. The measurements presented in this paper were taken with a single bunch of positrons.

\section{CESR SINGLE BUNCH DYNAMICS}

If collective effects are ignored, the standard deviation bunch length in a storage ring can be calculated from the synchrotron radiation integrals from [3]

$$
\sigma_{\tau}=\frac{\alpha}{\Omega_{\mathrm{s}}}\left(\frac{\sigma_{\varepsilon}}{\mathrm{E}_{0}}\right)=\frac{\alpha}{\Omega_{\mathrm{s}}} \sqrt{\mathrm{C}_{\mathrm{q}} \mathrm{E}_{0}^{2}\left(\frac{\mathrm{I}_{3}}{2 \mathrm{I}_{2}+\mathrm{I}_{4}}\right)}
$$

where $\mathrm{I}_{2}, \mathrm{I}_{3}$ and $\mathrm{I}_{4}$ are the synchrotron radiation integrals. The term $\alpha$ is the momentum compaction, $\sigma_{\varepsilon} / \mathrm{E}_{0}$ is the energy spread, $\Omega_{\mathrm{s}}$ is the synchrotron tune, $\mathrm{E}_{0}$ is the nominal energy, and $\mathrm{C}_{\mathrm{q}}$ is the constant of $3.84 \times 10^{-13} \mathrm{~m}$. The synchrotron integrals and longitudinal parameters that reflect CESR when the streak camera experiments were performed are denoted in table 1 .

\begin{tabular}{|c|c||c|c|}
\hline $\mathrm{I}_{1}$ & $8.528 \mathrm{~m}$ & RF Freq. & $499.765 \mathrm{MHz}$ \\
\hline $\mathrm{I}_{2}$ & $1.047 \times 10^{-1} \mathrm{~m}^{-1}$ & $\mathrm{U}_{0}$ & $1.1434 \mathrm{MeV}$ \\
\hline $\mathrm{I}_{3}$ & $2.300 \times 10^{-3} \mathrm{~m}^{-2}$ & $\sigma_{\mathrm{z}} / \mathrm{E}_{0}$ & $6.728 \times 10^{-4}$ \\
\hline $\mathrm{I}_{4}$ & $1.482 \times 10^{-3} \mathrm{~m}^{-1}$ & $\sigma_{\mathrm{z}}$ & $1.719 \times 10^{-2} \mathrm{~m}$ \\
\hline $\mathrm{I}_{5}$ & $5.092 \times 10^{-4} \mathrm{~m}^{-1}$ & $\alpha$ & $1.11 \times 10^{-2}$ \\
\hline Energy & $5.289 \mathrm{GeV}$ & $\mathrm{V}_{\mathrm{rf}}$ & $6.70 \mathrm{MV}$ \\
\hline
\end{tabular}

Table 1. The synchrotron integral and longitudinal parameters for CESR.

As the intensity of the bunch increases, collective effects that modify the slope of the RF wave have to be included. Collective effects lead to changes in the bunch distribution. Two collective effects examined in the past on CESR are potential well distortion and single bunch coherent instabilities. Past measurements with the NRF cavities present indicate that potential well distortion is the main single bunch longitudinal collective effect in CESR[2]. During these measurements no single bunch coherent instability was registered, up to the highest value of current allowed, with the NRF or SRF cavities. 
One of the goals of this paper is to determine the change in the bunch distribution with current (potential well distortion) due to the vacuum chamber impedance, and compare these distributions with the simulated distributions. We use the general formalism of Vlasov's Theory for calculating the distribution of particles in CESR. Under the assumption that the energy distribution remains unchanged, the steady state longitudinal distribution, $\psi(\tau)$, is a function of the resistance $(\mathrm{R})$, capacitance $(\mathrm{C})$, and inductance $(\mathrm{L})$ parts of the vacuum chamber impedance as[4,5]:

$$
\begin{aligned}
& \frac{\partial \psi(\tau)}{\partial \tau}=\frac{-\mathrm{eE}_{0} \psi(\tau)}{\sigma_{\varepsilon}^{2} \alpha \mathrm{T}_{0}} \times \\
& {\left[\frac{\mathrm{V}_{\mathrm{rf}} \cos (\omega \tau+\varphi)-\mathrm{QR} \psi(\tau)+\frac{\mathrm{Q}}{\mathrm{C}} \int_{-\infty}^{\tau} \mathrm{d} \tau^{\prime} \psi\left(\tau^{\prime}\right)-\mathrm{U}_{0}}{1+\frac{\mathrm{eE}_{0} \mathrm{QL} \psi(\tau)}{\sigma_{\varepsilon}^{2} \alpha \mathrm{T}_{0}}}\right] .}
\end{aligned}
$$

The longitudinal distribution, $\psi(\tau)$, can be determined by numerical integration of the above equation. These simulated distributions can be fit to the measured bunch distribution to determine the vacuum chamber impedance in CESR.

\section{STREAK CAMERA BUNCH DISTRIBUTIONS AND MEASUREMENTS}

Streak camera profiles of the bunch distribution are 512 pixels in size. Each profile is fit to a function that characterizes the distribution of the bunch. An asymmetric Gaussian function adequately characterizes the bunch shape in CESR and is given by

$$
I(z)=I_{0}+I_{1} \exp \left\{-\frac{1}{2}\left(\frac{(z-\bar{z})}{(1+\operatorname{sgn}(z-\bar{z}) A) \sigma}\right)^{2}\right\}
$$

where $I_{0}=$ pedestal, $I_{1}=$ peak of the asymmetric Gaussian. Figure 1 is an example of a CESR bunch distribution profile fit to an asymmetric Gaussian function. The fit determines the mean $\bar{z}$, asymmetry factor A, background level $\mathrm{I}_{0}$, peak of the asymmetric Gaussian $\mathrm{I}_{1}$, and width $\sigma$ of the distribution.

\subsection{Single Bunch Low Current Measurements}

The low current bunch length is computed from the synchrotron frequency, energy spread, and momentum compaction. The momentum compaction and energy spread are determined from CESR's lattice functions (table 1). The synchrotron frequency was measured with a spectrum analyzer to be $20.7 \mathrm{kHz}$. This corresponds to a low current bunch length of 57.3ps (17.2mm).

The longitudinal distribution at low current, with minimized collective effects, allows comparisons between the CESR model and the time calibration of the streak camera to be made. The low current positron bunch distribution was measured by taking 10 streak camera pictures with a single bunch present in CESR. The low current bunch length was measured to be $17.3 \mathrm{~mm}$.

The low current measurements show that the measured bunch length (without resolution correction) is $0.5 \%$ larger than the theoretical bunch length. If a resolution correction of $1.73 \mathrm{~mm}(5.77 \mathrm{ps})$ is subtracted in quadrature, the measured and theoretical bunch lengths agree. From previous studies of this camera systematic errors, a resolution correction of $1.73 \mathrm{~mm}$ is within reason [6].

\subsection{Bunch length as a Function of Current}

The CESR longitudinal bunch distribution between the currents of 0.5 to $18 \mathrm{~mA}$ was measured with a single bunch present. At each current setting, ten streak camera pictures of the bunch distribution were taken. Each picture was fit to an asymmetric Gaussian function and the mean bunch length and asymmetry factor was computed. The mean bunch length and asymmetry factor as a function of current is shown in figure 2 (a) and (b).

There are several noteworthy features of the experimental data: 1) The bunch length increases linearly with current, as denoted by the fit to the data in figure 2(a). A $0.104 \mathrm{~mm} / \mathrm{mA}$ growth rate in the bunch length is observed over the range of currents measured. A growth rate of $0.065 \mathrm{~mm} / \mathrm{mA}$ was measured with the NRF cavities present [2]. This increase in growth rate can be attributed to an increase in the inductive part of the impedance and/or a decrease in the capacitive part of the impedance.
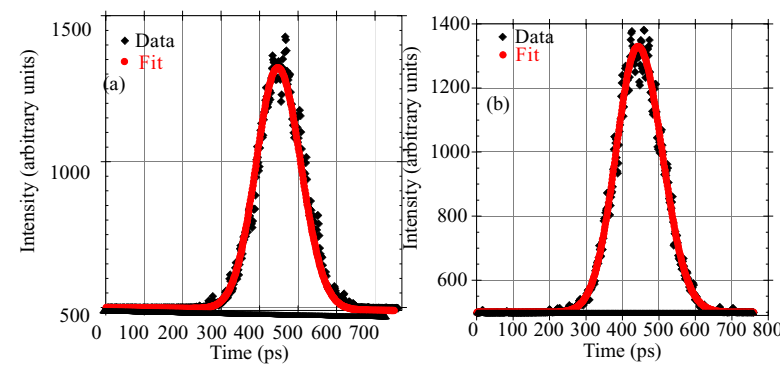

Figure 1 (color). A single streak camera picture of the CESR bunch distribution at a single bunch current of (a) $3 \mathrm{~mA}$, and (b) $18 \mathrm{~mA}$.

2) The asymmetry factor also increases linearly as a function of current. A distribution with a negative asymmetry factor has a larger tail than head. With increasing current, the tail of the distribution gets longer, which is a signature of potential well distortion due to the resistive part of the vacuum chamber impedance. These distributions, like the ones displayed in figure 1 , can be used to extract the vacuum chamber impedance of CESR. The growth rate of the asymmetry factor with the SRF cavities is $-0.0042 \mathrm{AF} / \mathrm{mA}$ and with the NRF cavities it was $-0.0062 \mathrm{AF} / \mathrm{mA}[2]$, indicating a reduction in the resistive part of the vacuum chamber impedance with the SRF cavities.

\subsection{CESR vacuum chamber impedance}

We model the CESR vacuum chamber impedance by only its resistive and inductive components 
(see point 2 below). The vacuum chamber impedance with the NRF cavities present was determined to be $\mathrm{R}_{\mathrm{NRF}}=1322 \pm 310 \Omega$ and $\mathrm{L}_{\mathrm{NRF}}=72 \pm 13 \mathrm{nH}$. One would expect that with the installation of the SRF cavities that the overall vacuum chamber impedance has been reduced and the measured bunch distributions should be able to confirm this statement. Simulated bunch distributions are provided by numerically integrating equation 1 .

As with the previous analysis of the vacuum chamber impedance, we assume that the resistance and inductance are constant over the measured range of bunch lengths. The impedance is determined from a $\chi^{2}$ fit between the simulated and the measured bunch distributions. From the $\chi^{2}$ fit to the data, the resistance for the CESR ring is $\mathrm{R}_{\mathrm{SRF}}=1016 \pm 348 \Omega$ and $\mathrm{L}_{\mathrm{SRF}}=96 \pm 24 \mathrm{nH}$. The uncertainties are determined by varying the simulation over the range of measurement errors on the asymmetry factor and rms width. Comparisons between the simulated bunch distributions using the above resistance and inductance and the streak camera measurements are shown in figure 2 (a) and (b).
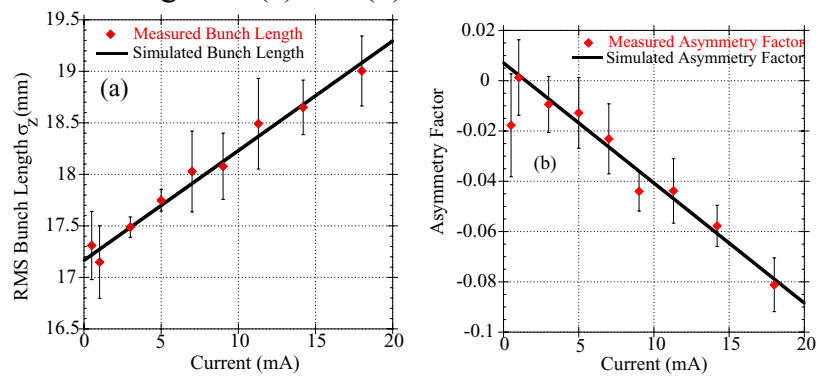

Figure 2 (color). The measured and simulated (a) bunch length and (b) asymmetry as a function of current.

There are several noteworthy features of the analysis:

(1) The resistive part of the vacuum chamber impedance can also be determined from Higher Order Mode measurements [7]. This measurement gives a resistive impedance of $R=939 \Omega$ with the $S R F$ cavities present in CESR. This independent measurement confirms our results and claim that the resistive impedance has been reduced with the SRF cavities.

(2) Since both inductive and capacitive parts of the vacuum chamber impedance affect primarily the bunch length (respectively lengthening and shortening) [2], differentiating between an increase in inductance and a decrease in capacitance is quite difficult. The simple model of the vacuum chamber impedance, which ignores the capacitive part, provides a reasonable picture of the measurements. Any capacitive part will likely be exhibited as a change in the inductive part of the impedance. A comparison of the impedance of CESR with the two different cavities shows that the resistive impedance has been reduced and the inductive impedance increased due to the SRF cavities. This increase in the inductive part is overstated in actuality; it is most likely a slight increase in the inductive part and a reduction in the capacitive part of the impedance. After all, the capacitive part of the impedance results from high shunt impedance cavities, such as the NRF cavities, and CESR used to have twenty NRF cavities and now only has four SRF cavities.

\section{CONCLUSIONS}

Measurements of the bunch distribution in CESR show that potential well distortion leads to some asymmetry and lengthening of the beam distribution at high current and that no single bunch coherent instability was apparent. From the measured bunch distributions, we were able to determine vacuum chamber impedance, by modeling it as a sum of the inductive and resistive parts. By comparing the measured bunch length dependence on the current with simulations, the vacuum chamber impedance was determined to have a resistance of $1016 \pm 348 \Omega$ and an inductance of $96 \pm 24 \mathrm{nH}$.

The low current single beam bunch length measurements confirmed our understanding of the streak camera and validated our theoretical model of CESR. Comparing previous bunch distribution measurements with the NRF cavities in CESR, the new SRF cavities have reduced the resistive part and increased the capacitive part of the CESR impedance. This change in impedance, as well as increased damping of higher order modes, has reduced the threshold of the longitudinal dipole-mode coupled bunch longitudinal instability, which, along with longitudinal feedback, has allowed higher current and higher luminosity running for CESR.

\section{ACKNOWLEDGMENTS}

The authors would like to thank Mike Billing and Mathew Stedinger for the simulation code.

\section{REFERENCES}

[1] Belomestnykh, S., et al., "Superconducting RF System for the CESR Luminosity Upgrade: Design, Status, Plans". SRF 960529-02.

[2] Holtzapple, R.L., et al., "Single Bunch Longitudinal Measurements at the Cornell ElectronPositron Storage Ring", Phys. Rev. ST Accel Beams 3, 034401, 2000.

[3] Helm, R. H., et al., "Evaluation of Synchrotron Radiation Integrals", SLAC, SLAC-PUB-1193, March 1973. 2pp.

[4] Haissinski, J., "Exact Longitudinal Equilibrium Distribution of Stored Electrons in the Presence of SelfFields," Il Nuovo Cimento, Vol. 18B, N.1, 11 November 1973, p. 72.

[5] Billing, M., "Bunch Lengthening via Vlasov Theory", CBN 80-2, 1980, 15pp.

[6] Holtzapple, R.L., "Experimental Techniques for the CESR Streak Camera", CBN01- 2, 2001. 9pp.

[7] Billing, M., Private Communication. 